\title{
Classification of Nuclear Reactor Severe Accidents using Probabilistic Neural Network based on Particle Swarm Optimization
}

Yoyok Dwi Setyo Pambudi

Center for Nuclear Reactor Technology and Safety, National Nuclear Energy Agency of Indonesi, Kawasan Puspiptek Gd. 80, Tangerang Selatan 15310, Indonesia

\section{ARTICLE INFO}

Article history:

Received: 17 February 2021

Received in revised form: 21 August 2021

Accepted: 23 August 2021

Keywords:

Probabilistic Neural Network Particle Swarm Optimization

Severe accident

Nuclear reactor

\begin{abstract}
A B S T R A C T
Due to its exposure to hazard and complexity, the identification and prediction of severe accident scenarios against an initiating event of a nuclear power plant remain a challenging task. This paper aims to classify severe accidents at the Advanced Power Reactor $1400 \mathrm{MWe}$ (APR1400), which include the loss of coolant accident (LOCA), total loss of feedwater (TLOFW), steam generator tube rupture (SGTR), and station blackout (SBO) using a standard Probabilistic Neural Network (PNN) and Particle Swarm Optimization-based Probabilistic Neural Network (PSO PNN). The algorithm has been implemented in MATLAB. The experiment results showed that supervised PNN PSO could classify severe accident of nuclear power plant by 19.4-point percent better than the standard PNN.
\end{abstract}

(c) 2021 Tri Dasa Mega. All rights reserved.

\section{INTRODUCTION}

As a complex and large system, nuclear power plant has a high probability to endure a fault and potentially damaged if severe accident were to happen. Among the potential fault in nuclear power plant are the disruption of the heat removal of the reactor cooling system and radioactive release. The failure of resolving these issues will undoubtedly cause a severe accident. A severe accident at a nuclear reactor is a form of accident that will result in damage to the core due to overheating. This occurs when the heat generated by the nuclear reactor core exceeds the

\footnotetext{
* Corresponding author

Email : dwisetyo@batan.go.id

DOI : $10.17146 /$ tdm.2021.23.3.6247
}

heat released by the cooling system due to a failure of the cooling system, as a result of leakage or loss of flow. So that, the core temperature exceeds the melting point at least in one nuclear fuel element.

Nuclear reactor operators attempt to distinguish accidents by identifying the transient patterns of significant parameters to classify the condition of nuclear power plant after the accident occurrs. Since there are hundreds of instruments that display typical patterns of transients in the reactor, operator may not be able to predict the major scenario of severe accident accurately. In order to manage severe accident, it is important to classify the initiating events, such as loss of coolant accident (LOCA), total loss of feedwater (TLOFW), steam generator tube rupture (SGTR), and station blackout (SBO). The knowledge of this initiating event is extremely important for the operators to be able to manage accidents, since the safety performance does not rely only on technical matters but also on human performance of nuclear power plant operator[1]. 
Failure of detecting and managing the initial event will cause the residual heat to damage or even melt the reactor core. Following this event, hydrogen will be generated in the reactor, which can potentially cause explosion.

For many years, the implementation of artificial intelligence has been commonly used in nuclear engineering areas such as diagnostics[2, 3], anomalies detection[4, 5], and signal validation[6]. Artificial neural networks (ANNs) capability of modeling complex systems without demanding an explicit knowledge among the variables becomes its strength over other approaches. The probabilistic neural network (PNN) is a neural network implementation of a Bayesian classifier that takes relative similarity of events and distinguish transients with identical signal forms.

Owing to the simplicity of training, PNN becomes a viable device for tackling many classifications and approximation problems [7 -9]. In nuclear area, PNN has been utilized for detection component and instrumentation diagrams of nuclear power plant[10], monitoring of nuclear power plant condition[11, 12], and rapid source term prediction[13].

PNN is designed to be straightforward and does not depend on training. However, standard PNN often requires all neuron in the training class to become redundant, and it can cause a large structure, which results in high computation time. Smoothing parameter plays an important role in the PNN, in order to make its classification capability better.

In relation to nuclear power plant, the classification of an initiating severe accident by using a standard PNN has been previously made. However, the data used for testing is limited and the recognition rate was not mentioned. This paper aims to classify the initiating event of severe accident nuclear power plant, such as LOCA, TLOFW, SBO, and SGTR, by using supervised PNN. The PNN was supervised using Particle Swarm Optimization (PSO) algorithm. PSO is a heuristic and real coded algorithm, andwas originally credited to Kennedy and Eberhart[14]. Primarily, it was intended to simulate social behaviour. In nuclear area, PSO has been used to investigate to the nuclear reactor reload optimization problem [15], PWR power distribution flattening, and critical heat flux prediction[16]. Combination of backpropagation neural networks and PSOhas been investigated in development of nuclear reactor control[17].

\section{SEVERE ACCIDENT OF PWR REACTOR.}

The Advanced Power Reactor 1400 (APR1400) is apressurized water reactorthat uses two coolant loops (primary and secondary loop). Primary loop is arranged as two closed loops connected in parallel to the nuclear reactor vessel. Each loop consists of two reactor coolant pumps, a steam generator, a $1.07 \mathrm{~m}$ ID hot leg pipe and 0.76 $\mathrm{m}$ ID cold leg pipes, and a pressurizer. The isometric view of APR1400 reactor was shown in Figure. 1. Primary loop removes the heat generated by fission reaction in the reactor core at high pressure. Secondary loop receives heat from the primary loop in the steam generator,generate the steam, and transfer the energy to generate electricity. Heat generated from the reactor core must always be removed by the reactor coolant system. If accident occurs, then heat removal will be disrupted. The heat from reactor core is still generated by reactions from delayed neutron although reactor core was already shutdown. This residual heat needs to be removed from the core by reactor cooling system. Failure of heat removal would cause a reactor accident. An accident such as LOCA is caused by leakage or rupture of primary loop pipe e.q. hot leg pipe and cold leg pipe. This leakage could be affected by pipe corrosion or broken due to earthquake.

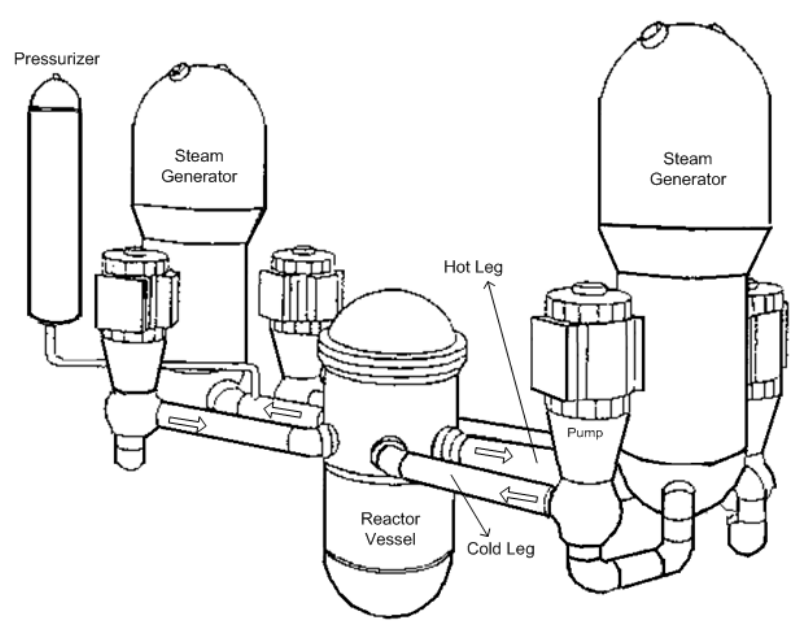

Fig. 1. APR1400 isometric view

For this work, severe accident scenarios of the reactor that would be identified are hot leg LOCA, cold leg LOCA, TLOFW, SGTR, and SBO. The data of severe accident scenarios of APR1400 was classified into five conditions. Original data was postulated and generated by using MAAP4 code, because it is difficult to get real data for severe accident of nuclear reactor. The feature space of the data is two dimensions e.q. break size and scram 
time. Some training samples for work 1 are shown in Table 1.

Table 1. Severe Accident Event (sample)

\begin{tabular}{|c|c|c|}
\hline Break size $\left(\mathrm{m}^{2}\right)$ & Scram time (s) & Classification \\
\hline 0.00051 & 917.94 & 1 \\
\hline 0.00079 & 602.84 & 1 \\
\hline 0.00114 & 398.38 & 1 \\
\hline 0.00155 & 282.76 & 1 \\
\hline 0.00203 & 206.04 & 1 \\
\hline 0.00257 & 158.09 & 1 \\
\hline 0.00535 & 73.163 & 1 \\
\hline 0.00051 & 583.02 & 2 \\
\hline 0.00079 & 351.11 & 2 \\
\hline 0.00114 & 228.50 & 2 \\
\hline 0.00155 & 165.14 & 2 \\
\hline 0.00317 & 77.949 & 2 \\
\hline 0.00383 & 64.398 & 2 \\
\hline 0.00383 & 64.298 & 3 \\
\hline 0.00045 & 45.792 & 3 \\
\hline 0.00090 & 45.880 & 3 \\
\hline 0.00180 & 45.25 & 3 \\
\hline 0.00000 & 46.3079 & 4 \\
\hline 0.00000 & 0.001 & 5 \\
\hline 0.00000 & 0.015 & 5 \\
\hline 0.00000 & 0.002 & 5 \\
\hline
\end{tabular}

Note: 1: Hot leg LOCA 2: Cold Leg LOCA 3: Steam Generator Rupture 4: Total Loss of Flow Accident 5: Station Black Out

For the SBO and TLOFW accidents, the value of break size of coolant pipe is zero, because in this scenario there is no break/rupture in water circulation pipe of the reactor. SBO is a condition when the power supply for reactor immediately shuts down. This condition makes control rod drop (SCRAM) automatically. TLOFW is a loss of water flow in primary loop that can be caused of the drop of all cooling pumps. For testing the proposed method, data from this work was generated at random in range $95 \%$ to $100 \%$ of the original data. Data generation is done to obtain a variety of hundreds of data, because in reality, physical measurements have an error precision about $\pm 5 \%$. This data generation is also used to test the algorithm with variations of data. After generation, the total data of severe accident event of the reactor become 1000 data. This data consists of 200 data of hot-leg LOCA, 200 cold-leg LOCA, 200 data of TLOFW, 200 data of SGTR accident, and 200 data of SBO. To examine the event classification by the neural network, the data are separated into training data and verification/testing data. The training data are used to train the neural network and verification data are used to independently examine it.

Data was normalized by $\mathrm{z}$-score, before processing data with algorithm. The formula of $z-$ score can be written as follow.

$$
x_{i n}=\frac{x_{i n}-\overline{x_{i n}}}{\tau_{x_{i n}}}
$$

Where $x_{i n}$ is input data, $\overline{x_{i n}}$ is mean of data, and $\tau_{x_{i n}}$ is standard deviation.

\section{METHODOLOGY}

\subsection{Supervised Probabilistic Neural Network (PNN)}

This work proposes a supervised PNN structure using PSO to find appropriate smoothing parameter of PNN. PNN It was first introduced by Specht[13], PNN was generally used for pattern classification problems. PNN network was trained by assigning each training neuron to a pattern unit according to its class. The advantage of PNN is that training is simple and momentary. PNN consist of four-layer neural network i.e., input unit, pattern unit, summation unit, and output unit layer. PNN architecture was shown in Figure 2.

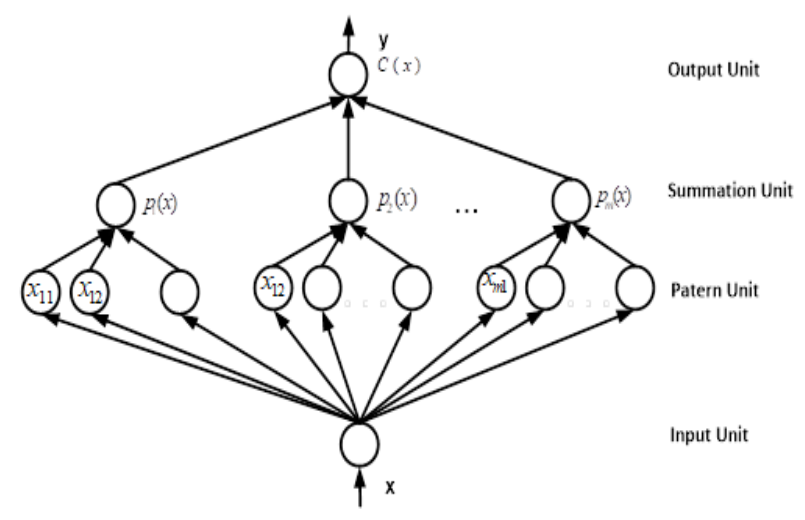

Fig. 2. Probabilistic neural network architecture

The input layer just merely distributes the input to the neurons without any calculation. Pattern layer calculates while receiving $x$ from input layer.

$$
\varphi_{i j}(x)=\frac{1}{\sigma^{d}(2 \pi)^{d / 2}} \exp \left[-\frac{\left(x-x_{i j}\right)^{T}\left(x-x_{i j}\right)}{2 \sigma^{2}}\right]
$$

Where $\varphi_{i j}$ is pattern layer value, $x$ is neuron input, $d$ is dimension of the pattern vector, $\sigma$ is the smoothing parameter and $x_{i j}$ is the class neuron vector. For a single population the probability density function $p_{i j}(x)$ is calculated by. 
$p_{i j}(x)=\frac{1}{\sigma^{d}(2 \pi)^{d / 2}} \frac{1}{N_{i}} \sum_{j=1}^{N_{i}} \exp \left[-\frac{\left(x-x_{i j}\right)^{T}\left(x-x_{i j}\right)}{2 \sigma^{2}}\right]$

where $N_{i}$ express the total number of samples in class. The summation layer computes the maximum similarity of pattern $x$ being classified into $C_{i}$ by summarizing and averaging the output of all neurons that have a place with a similar class.

$$
C(x)=\operatorname{argmax}\left\{\varphi_{i j}(x)\right\} \quad i=1,2,3 \ldots \ldots, m
$$

where $C(x)$ denotes the approximated class $x$ of the pattern and $m$ is the total number of classes in the training samples.

\subsection{Particle swarm optimization for smoothing parameter optimization}

PSO is one of the current heuristic algorithms. It was created through simulation of abridged social environment, and has been found to be firm in clarifying nonlinear optimization problems. PSO can deliver solutions with stable convergence characteristic at a high speed. The optimization technique of PSO was developed based on the behavior of a group of insects or birds. Any individual or particle act in a dispersed way by using their own intelligence and also influenced the behavior of the cumulative group. Thus, if a particle or a bird discovers the right path or short parth heading to the food source, the rest of the group will be able to follow the path soon despite their own location in the group. The basic particle position and velocity update equation can be represented as Eq. 5.

$$
\begin{gathered}
V_{j}(i)=V_{j}(i-1)+c_{1} \times r_{1} \times\left[P_{\text {best }}-X_{j}(i-1)\right]+c_{2} \\
\quad \times r_{2} \times\left\{G_{\text {best }}-X_{j}(i-1)\right] \\
X_{j}(i)=X_{j}(i-1)+V_{j}(i) \\
j=1,2,3, \ldots N \mathrm{j}=1,2,3, \ldots \mathrm{N}
\end{gathered}
$$

Where $V_{j}$ is velocity of particle $\mathrm{i}$ at iteration $\mathrm{j}, c_{1}$ , $c_{2}$ is positive values of acceleration coefficient, $r_{1}, \quad r_{2}$ is random number between 0 and $1, P_{b e s t}$ is best location of each particle, $G_{b e s t}$ is best location of neighbors, and $X_{j}$ is velocity of particle $\mathrm{i}$.

Original PSO has the disadvantage that the speed is excessively fast so that the minimum value of the objective function is often passed. The improvement of PSO were done by addition of an inertial weight $(w)$ to reduce velocity speed. Inertia weight is updated using Eq. 9. Therefore, with increasing iteration, the inertial weight will linearly be decreased.

$$
\begin{aligned}
& V_{j}(i)=w \times V_{j}(i-1)+c_{1} \times r_{1} \times\left[P_{\text {best }}-x_{j}(i-1)\right]+ \\
& c_{2} \times r_{2} \times\left\{G_{\text {best }}-x_{j}(i-1)\right] \\
& c_{1}=c_{1 \text { max }}-\left(\frac{c_{1 \text { max }}-c_{1 \text { min }}}{K}\right) k
\end{aligned}
$$

where $k$ is iteration number

$$
\begin{aligned}
& c_{2}=c_{1 \min }+c_{1 \max }-c_{1} \\
& w(i)=w_{\max }-\left(\frac{w_{\max }-w_{\min }}{i_{\max }}\right)
\end{aligned}
$$

During the PSO training, the particle which has the inferior position is found and replaced with the best position particle in the swarm [18]. This modification is done by inserting the coefficient c3 into Eq. 5 and the equation become Eq. 10.

$$
X_{j}(i)=X_{j}(i-1)+c_{3} \times V_{j}(i)
$$

While the value of the coefficient $c_{3}$ was chosen randomly in the range $[0.1,0.5]$. This work was carried out in the Matlab environment. An algorithm of modified PSO to acquire optimal smoothing factor of PNN given in Figure 3 as following:

\begin{tabular}{|l|} 
Begin \\
FOR each Particle \\
Initialize Particle \\
End \\
FOR each Particle \\
Determine fitness function by particle i.e., number of miss \\
recognition rate of PNN. \\
If fitness value is exceeding than the best fitness in history \\
Set current value as the new best particle \\
update its position (i.e., smoothing factor) \\
WHILE maximum iterations or measures accomplished, then \\
stop the iteration \\
End.
\end{tabular}

Fig. 3. Algorithm code of PSO PNN

\section{RESULT AND DISCUSSION}

Computer work conducted to determine the performance comparison between standard and supervised PNN. At first, standard PNN was used to classify severe accident nuclear reactor. Before running the PNN algorithm, the smoothing parameter should be inputted. The value of optimal smoothing should be chosen less than the standard deviation of the observed data. The smoothing parameter was frequently chosen at small or large number[19]. Based on this criterion, the value of 
smoothing parameter for standard PNN was chosen to be 0.1 and 1 .

The proposed calculation was tried by utilizing the consistent data set which was generated by using MAAP4 code. In PNN we called a training data as a class neuron and testing data as testing neuron. For this experiment the number of each class neuron and testing neuron was chosen in a combination 50-150, 50-200, 100-100, and 100200. The recognition rate result for standard PNN algorithm is shown at Table 2 .

Table 2. Recognition rate result of standard PNN algorithm

\begin{tabular}{ccccc}
\hline & \multicolumn{4}{c}{ Class \& Test Neurons Combination } \\
\cline { 2 - 5 } & $50-150$ & $50-200$ & $100-100$ & $100-200$ \\
\hline$\sigma=0.1$ & $75.5 \%$ & $76.5 \%$ & $76.8 \%$ & $76.8 \%$ \\
$\sigma=1$ & $64.53 \%$ & $64.4 \%$ & $64.40 \%$ & $64.60 \%$ \\
\hline
\end{tabular}

The recognition rate for combination $50-150$ is $64.3 \%$ for smoothing parameter $(\sigma)$ was set 1 and $75.5 \%$ if smoothing parameter was set in 0.1 . The highest recognition rate was $76.8 \%$ for $\sigma=$ 0.1 and $64.60 \%$ for $\sigma=0.1$, theywere obtained in the combination of class 100 neurons and 200 class neurons tests. This mean that all data is used as class sources. The result indicating that the lower smoothing parameter could give the higher recognition rate.

To find appropriate smoothing parameter of PNN, initial number iteration for PSO was set 50, number of swarms was 30, boundary of smoothing parameter was set in the range of $[-10,10]$. Inertial weight max is 0.9 and weight $\min$ is 0.4 . Parameter for optimized PNN PSO is shown in Table 3.

Table 3. Parameter for Optimization PNN PSO

\begin{tabular}{lc}
\hline Parameter & Value \\
\hline \hline Iteration number $(\mathrm{K})$ & 50 \\
Particle swarm size $(\mathrm{S})$ & 30 \\
Minimum first confidence & 1.5 \\
coefficient $\left(c_{1 \min }\right)$ & \\
Maximum first confidence & 2.5 \\
coefficient $\left(c_{2 \max }\right)$ & 0.4 \\
Minimum inertia $\left(w_{\min }\right)$ & 0.9 \\
Maximum inertia $\left(w_{\max }\right)$ & \\
\hline
\end{tabular}

The recognition result of Supervised PNN (PNN PSO) algorithm for this work was shown in Table 4.
Table 4. Recognition rate result of PNN PSO algorithm

\begin{tabular}{lrrrr}
\hline & \multicolumn{4}{c}{ Class \& Test Neurons Combination } \\
\cline { 2 - 5 } & $50-150$ & $50-200$ & $100-100$ & $100-200$ \\
\hline $\begin{array}{l}\text { Recognition } \\
\text { rate }\end{array}$ & $92.4 \%$ & $95.3 \%$ & $93.4 \%$ & $96.5 \%$ \\
$\begin{array}{l}\text { Smoothing } \\
\text { parameter }\end{array}$ & 0.000328 & 0.0503 & 0.0020 & 0.0012 \\
\hline
\end{tabular}

The largest recognition rate of $96.5 \%$ was obtained in combination of 100 class neurons and 200 test neurons. The smoothing value of this combination is 0.0012 . Meanwhile the combination 50-150 has a less recognition rate of about $92.4 \%$ using smoothing parameter of 0.00038 . It can be shown from the work that standard PNN using $\sigma=0.1$ achieves $76.40 \%$ averaged recognition rate. Meanwhile, supervised PNN using PSO could achieve $94.40 \%$ averaged recognition rate. This result shows that the combination of 100-200 could obtain the best result of the recognition rate.

The reason is the attachment PSO algorithm on PNN could find appropriate smoothing parameter for the classification. Compared to the standard PNN, The proposed PNN PSO is better by 19.4point percent.

After several training and testing, the proposed PNN-based PSO algorithm could classify five kinds of accidents on nuclear reactors such as hot leg LOCA, cold leg LOCA, SGTR, TLOFW and SBO using the reactor scram time and break size data.

The scram time is the short time integration of some selected signals immediately after reactor scram, and break size of pipe tube in reactor.

\section{CONCLUSION}

This paper presented supervised probabilistic neural network based on particle swarm optimization for classifying the initiating events under severe accident of nuclear power plant. The work shows that standard PNN achieves $76.40 \%$ averaged recognition rate, while supervised PNN by PSO could achieve $94.40 \%$ averaged recognition rate. The largest recognition rate of $96.5 \%$ was obtained in a combination of 100 class neuron and 200 test neurons. These results suggest that the PNN optimized by PSO could precisely allocate a lot of data of represented accident in AP1400 reactor like hot leg LOCA, cold leg LOCA, SGTR, TLOFW, and SBO.

\section{ACKNOWLEDGMENT}

This research was upheld and funded by the Nuclear Research \& Development Program (DIPA 2021) at the Centre for Nuclear Reactor Technology and Safety - BATAN. 


\section{AUTHOR CONTRIBUTION}

Yoyok Dwi Setyo Pambudi is the main contributor who took part in planning, conducting and reporting this paper. The author read and approved the final version of the paper.

\section{REFERENCES}

1. Santoso S., Himawan R., Situmorang J., Suryono T.J., Edison E. Reactor Operational Experience Review and Analysis Based on Un-intended Reactor Trip Data. J. Teknol. Reakt. Nukl. TRI DASA MEGA. 2019. 21(2):71-78.

2. No Y.-G., Kim J.-H., Na M.-G., Lim D.-H., Ahn K.-I. Monitoring Severe Accidents Using AI Techniques. Nucl. Eng. Technol. 2012. 44(4):393-404.

3. Kim S.H., Shin S.G., Han S., Kim M.H., Pyeon C.H. Feasibility Study on Application of an Artificial Neural Network for Automatic Design of a Reactor Core at the Kyoto University Critical Assembly. Prog. Nucl. Energy. 2020. 119:103183.

4. Zhao Y., Li T., Zhang X., Zhang C. Artificial Intelligence-based Fault Detection and Diagnosis Methods for Building Energy Systems: Advantages, Challenges and the Future. Renew. Sustain. Energy Rev. 2019. 109:85-101.

5. Tian D., Deng J., Vinod G., Santhosh T. V, Tawfik H. A Constraint-based Genetic Algorithm for Optimizing Neural Network Architectures for Detection of Loss of Coolant Accidents of Nuclear Power Plants. Neurocomputing. 2018. 322:102-109.

6. Lee D., Seong P.H., Kim J. Autonomous Operation Algorithm for Safety Systems of Nuclear Power Plants by Using Long-short Term Memory and Function-based Hierarchical Framework. Ann. Nucl. Energy. 2018. 119:287-299.

7. Shi J., Deng Y., Wang Z. Analog Circuit Fault Diagnosis Based on Density Peaks Clustering and Dynamic Weight Probabilistic Neural Network. Neurocomputing. 2020. 407:354365.

8. Santhosh T. V, Gopika V., Ghosh A.K., Fernandes B.G. An Approach for Reliability Prediction of Instrumentation \& Control Cables by Artificial Neural Networks and Weibull Theory for Probabilistic Safety Assessment of NPPs. Reliab. Eng. Syst. Saf. 2018. 170:31- 44.

9. Zhu H., Lu L., Yao J., Dai S., Hu Y. Fault
Diagnosis Approach for Photovoltaic Arrays Based on Unsupervised Sample Clustering and Probabilistic Neural Network Model. Sol. Energy. 2018. 176:395 -405.

10. Gao W., Zhao Y., Smidts C. Component Detection in Piping and Instrumentation Diagrams of Nuclear Power Plants Based on Neural Networks. Prog. Nucl. Energy. 2020. 128: 103491.

11. Liu J., Seraoui R., Vitelli V., Zio E. Nuclear Power Plant Components Condition Monitoring by Probabilistic Support Vector Machine. Ann. Nucl. Energy. 2013. 56:23-33.

12. Dos Santos M.C., Pinheiro V.H.C., Do Desterro F.S.M., De Avellar R.K., Schirru R., Dos Santos Nicolau A., et al. Deep Rectifier Neural Network Applied to the Accident Identification Problem in a PWR Nuclear Power Plant. Ann. Nucl. Energy. 2019. 133:400-408.

13. Zhao Y., Tong J., Zhang L. Rapid Source Term Prediction in Nuclear Power Plant Accidents Based on Dynamic Bayesian Networks and Probabilistic Risk Assessment. Ann. Nucl. Energy. 2021. 158:108217.

14. Norouzi N., Sadegh-Amalnick M., Alinaghiyan M. Evaluating of the Particle Swarm Optimization in a Periodic Vehicle Routing Problem. Measurement. 2015. 62:162169.

15. Augusto J.P. da S.C., Dos Santos Nicolau A., Schirru R. PSO with Dynamic Topology and Random Keys Method Applied to Nuclear Reactor Reload. Prog. Nucl. Energy. 2015. 83:191-196.

16. Jamalipour M., Gharib M., Sayareh R., Khoshahval F. PWR Power Distribution Flattening Using Quantum Particle Swarm Intelligence. Ann. Nucl. Energy. 2013. 56:143150.

17. Pambudi Y.D.S., Wahab W., Kusumoputro B. Particle Swarm Optimization-Based Direct Inverse Control for Controlling the Power Level of the Indonesian Multipurpose Reactor. Sci. Technol. Nucl. Install. 2016. 2016:1- 9.

18. Coban R. Power Level Control of the TRIGA Mark-II Research Reactor Using the Multifeedback Layer Neural Network and the Particle Swarm Optimization. Ann. Nucl. Energy. 2014. 69:260-266.

19. Jiang Y., Li X., Huang C., Wu X. Application of Particle Swarm Optimization Based on CHKS Smoothing Function for Solving Nonlinear Bilevel Programming Problem. Appl. Math. Comput. 2013. 219(9):4332 4339. 
Tri Dasa Mega Vol. 23 No. 3 (2021) 99-104 
Tri Dasa Mega Vol. 23 No. 3 (2021) 99-104 